\title{
AIAA-95-1045
}

\section{Using and Distributing Spaceflight Data: The Johnson Space Center Life Sciences Data Archive}

J. Buckey

UT-Southwestern Medical Center Houston, TX

J. Turner, T. White, J. Havelka

Martin Marietta Services

Houston, TX

J. Cardenas

NASA Johnson Space Center Houston, TX

\section{Life Sciences and Space Medicine Conference April 3-5, 1995 / Houston, TX}





\section{USING AND DISTRIBUTING SPACEFLIGHT DATA: THE JOHNSON SPACE CENTER LIFE SCIENCES DATA ARCHIVE}

J. C. Buckey M.D., UT-Southwestern Medical Center, J.N. Turner B.S., T.S. White B.S., J.A. Havelka B.S., Martin Marietta Services, J.A. Cardenas B.S., NASA-Johnson Space Center Houston, Texas

\section{ABSTRACT}

Life sciences data collected before, during and after spaceflight are valuable and often irreplaceable. The Johnson Space Center Life Sciences Data Archive has been designed to provide researchers, engineers, managers and educators interactive access to information about and data from human spaceflight experiments. The archive system consists of a Data Acquisition System, Database Management System, CD-ROM Mastering System and Catalog Information System (CIS). The catalog information system is the heart of the archive. The CIS provides detailed experiment descriptions (both written and as QuickTime movies), hardware descriptions, hardware images, documents, and data. An initial evaluation of the archive at a scientific meeting showed that $88 \%$ of those who evaluated the catalog would want to use the system when completed. The majority of the evaluators found the archive flexible, satisfying and easy to use. We conclude that the data archive effectively provides key life sciences data to interested users.

\section{INTRODUCTION}

Opportunities to study physiology in space are limited, which makes the data collected before, during and after spaceflight particularly valuable. Published reports focus on major findings and often provide only summarized results. Analyses across experiments and disciplines can be difficult to perform.

Early in the space program the importance of preserving data was recognized. After the Skylab program in 1974 an effort was made to archive the life sciences data from the program (11) and to provide analysis tools for the data (6). Computer technology at that time, however, was limited. The archive required a room to house the digital data tapes, microfilm, strip chart records and documents. Users had to travel to the

This paper is declared a work of the U.S. Government and is not subject to copyright protection in the United States archive and use the facilities provided. Today, the Skylab data resides at the Texas Medical Center Library in Houston, Texas. Detailed information about the experiments is hard to find, and much of the data (e.g. the analog tapes) can no longer be accessed. Only the microfilms and various documents remain for the interested researcher. Today the best single source of information on the Skylab program is a book (3), but this publication includes mainly summarized data.

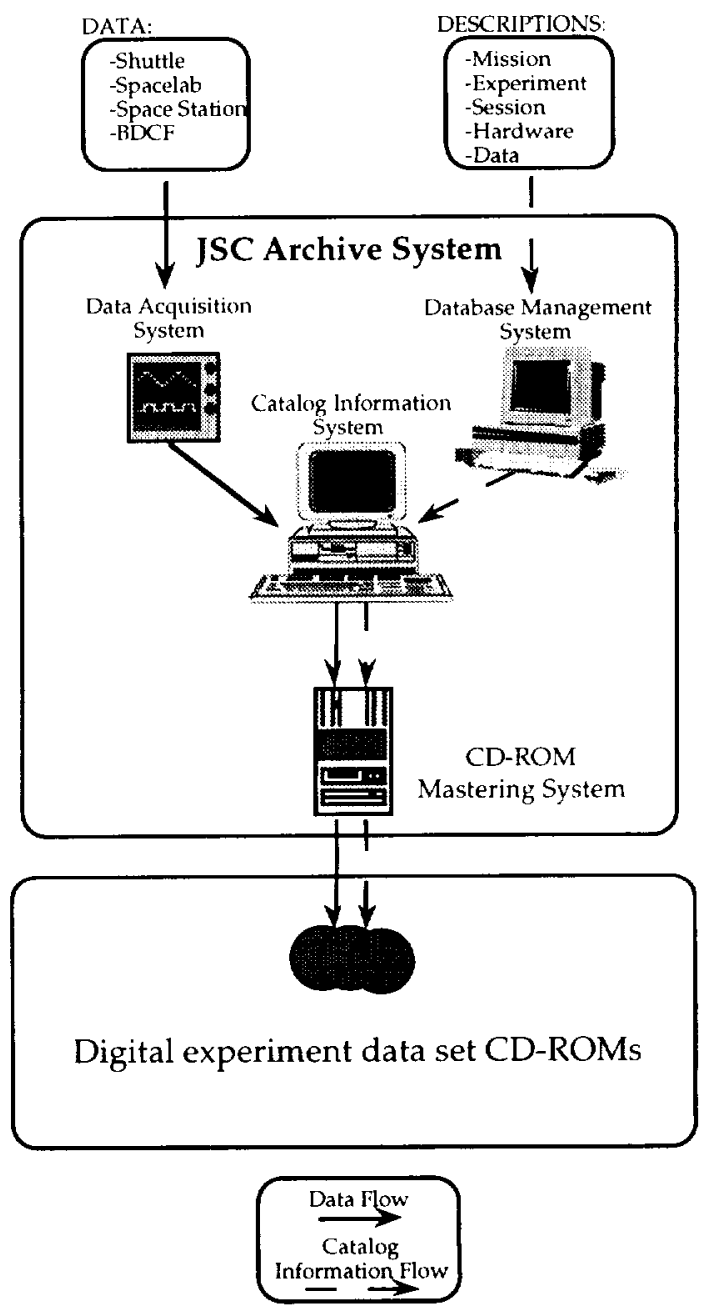

Figure 1. Overall organization of the archive. $\mathrm{BDCF}=$ Baseline Data Collection Facility .

The Spacelab program has produced a tremendous influx of new life sciences 
findings (12). To realize the full benefit of these data, an archive that allows interactive access to all the data is needed. The archive should have the capability to search across experiments, disciplines and missions. Also, new digital storage and retrieval technologies allow information that previously required a room for storage, to be kept on a CD-ROM.

The Johnson Space Center Life Sciences Data Archive has been designed to allow researchers, engineers, managers and educators easy access to information about and data from human experiments performed in space. At the Ames Research Center a similar effort exists to archive nonhuman data. Descriptive information from both archives will also be available via the Internet or modem connection at the National Space Science Data Center (NSSDC) as described previously (4). The actual human data will only be available on CD-ROM.

Currently, the Johnson Space Center Life Sciences Data Archive is using the Spacelab Life Sciences 1 flight information to create a prototype archive. This paper will present the design of the prototype as well as the results from the first evaluation.

\section{SYSTEM DESCRIPTION}

The archival system has four main components (Figure 1). The Data Acquisition System (DAS) takes data from a variety of sources (investigator labs, Shuttle telemetry) and puts them into a standard format for use in the archive. For example, a spaceflight experiment may have used an analog tape recorder for pre and post flight data collection. In flight, this experiments data may have been in the telemetry stream from the Orbiter. Information from both these sources can be read by the MIRAGE software $(1,9)$ used by the DAS. Once in the archive, these data would share a common format (ASCII) that could be used by a variety of commercially available software packages. Text is scanned into the DAS.
The Database Management System (DBMS) will be used in the future to track data from initial acquisition until final archival. This system is still in development, since current archive activity is focused on missions that have already flown. This system will be based on the Oracle client server relational database software.

Once all the data from a particular flight have been archived, the catalog for that flight will be placed on a CD-ROM. The CDROM Mastering System allows for CDROMs to be produced from the experiment data for distribution and deep archival.

The heart of the system is the Catalog Information System (CIS).

\section{CATALOG INFORMATION SYSTEM}

This system is based on commercially available FileMaker Pro database software. The CIS contains detailed descriptions of the mission, experiments, experiment sessions, hardware, personnel, and datasets. The CIS allows the user to search through the database to find information on particular parameters, experiments, subjects or hardware. Help screens are provided to guide the novice user.

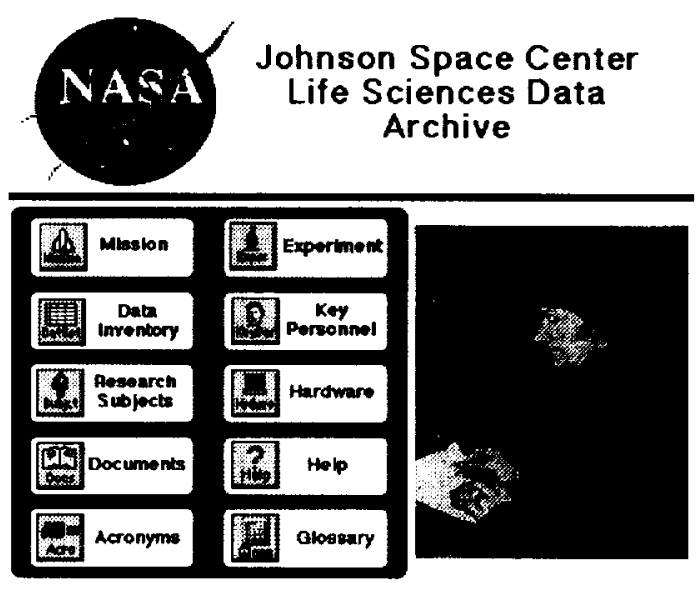

Figure 2. Entry screen to catalog information system.

Figure 2 shows the entry screen for the CIS. The user can press any of the software "buttons" and advance through the system. Figure 3 shows the screen that the user will encounter if the experiment button is 
pushed. The experiment information is contained on two pages in several fields. One of these fields contains a QuickTime movie that describes, at a lay level, what the experiment is about. A detailed, scientific description appears in the experiment description field. By using both video and text, the CIS can meet the needs of a variety of users. A casual user may find the detailed experiment description too scientific and difficult to understand, but the video would provide key information more simply. A serious researcher might find the video too superficial, so the experiment description would help this kind of user. References to the open scientific literature are provided for those who need further information.

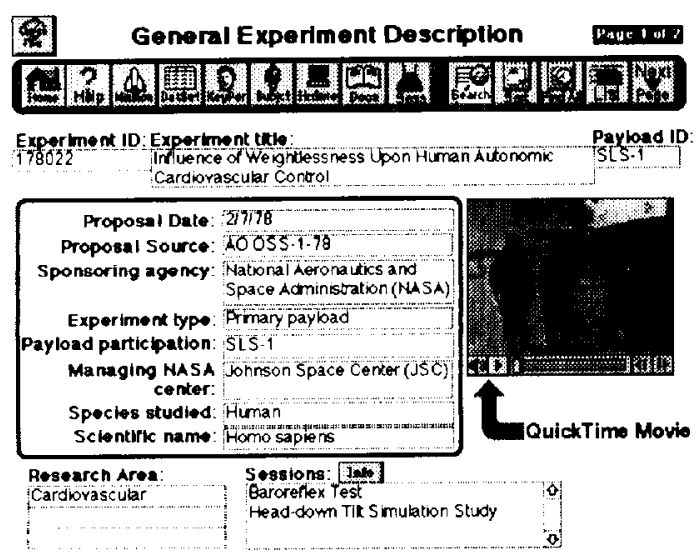

Figure 3. Typical archive information page. This page describes a particular experiment and includes a QuickTime movie.

Some of the fields available in the experiment description are: experiment ID, experiment title, payload ID, research area, parameters measured, session, experiment description, personnel (principal investigator, co-investigator, key personnel), publications, and hardware. These are all searchable fields. This means that a user can find experiments that share certain characteristics. If the user wanted all the experiments in cardiovascular physiology, for example, he/she could do a search using the research area field. All the experiments dealing with cardiovascular physiology would be found. Similarly, a search can be done to find all experiments that measured a particular parameter or used a particular piece of hardware.
The icon bar across the top of the page shown in figure 3 is common to all screens in the CIS. The user navigates through the system by pressing these buttons. Their functions are described below.

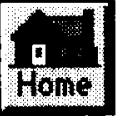
the opening page.

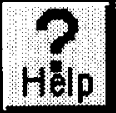

Help- This provides instruction on how to use the system. An introduction to navigating through the system is provided as an animated presentation. This is produced with Macromind director software. The user receives both animation and audio of how to navigate through the catalog system.

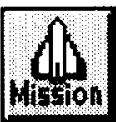

Mission- This takes the user to the mission page. This page gives both a text and video description of the mission, shows what experiments were on a given mission, and who the key personnel were.

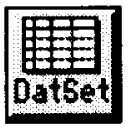
actual datasets. When this button is pressed within a particular experiment only the data from that experiment are displayed. The data set page has several fields to describe the dataset, i.e. what hardware was used, what the analysis techniques were, when and where the data were acquired. From the dataset page the user can press a button to see the data. Analyzed data are displayed using Microsoft Excel software. Continuous data are in ASCII format and can be displayed using a variety of software packages (Microsoft Excel, LabView, DADISP).

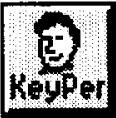

KeyPer- This takes the user to a page that provides the address, phone 
number and e-mail address of the key personnel involved in the mission. subjet Subjct- By pressing this button, the
user can find some key information about the people who participated in the experiments.

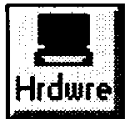

Hrdwre- This takes the user to descriptions of the hardware used. This database includes pre and post flight hardware, in addition to flight items. A written description and picture is included for each item.

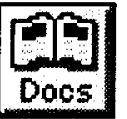

Docs- This takes the user to final reports, test plans, proposals, and work statements for the experiments. Scientific publications in the open literature are not listed here, but are included as references on the experiment page itself.

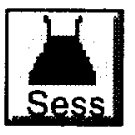

Sess- By pressing this button the user moves to descriptions of the major sessions that constituted an experiment. Many experiments have subdivisions. For example, a cardiovascular experiment may have stand tests, lower body negative pressure tests, and exercise tests all as part of the one experiment. Each one of these sessions may use different protocols or hardware, and measure different parameters. To provide an interested user with enough information to understand the experiment, they need a session page that describes what took place in an individual session.

\section{Searches}

Within each of the major sections of the catalog (experiments, sessions, datasets, hardware, documents, key personnel, subjects) the user can perform searches on the various fields. For example, if a user wanted to find all the datasets that included a particular parameter, he/she could search in the parameters measured field and find all the datasets that include this parameter. As the catalog grows in size this will be a particularly powerful feature. Boolean searches on multiple fields (e.g. a particular parameter and a particular experiment) can also be done.

\section{Portability}

The software for the archive will run on either Macintosh or PC based computers. To get the catalog, a user would order a CDROM with the catalog information system on it. This CD-ROM would include a run time version of the FileMaker Pro software; the user would need to have a CD-ROM reader and a spreadsheet program to view the data.

\section{Privacy}

The display of individual data from crewmembers who participated in experiments in space presents a special problem. These data are covered by the Privacy Act (8) and internal NASA regulations (5). The archive has to balance the requirement for NASA to disseminate space information $(7,10)$ with the need to maintain privacy. Different approaches to this problem exist (2). A specific policy for the archive is under development. For evaluation purposes, the actual data have been replaced with zeroes.

\section{EVALUATION}

To guide future development, the prototype data archive was displayed at the American Society for Gravitational and Space Biology (ASGSB) annual meeting in October 1994. This meeting attracts researchers working in space life sciences research. At this meeting attendees had the opportunity to use the archive. They could browse through the various pages, and evaluate how easy or hard it was for them to use the system. After using the system each person was encouraged to fill out a questionnaire. A total of 91 people used the archival system and filled out a questionnaire.

These questionnaire results provide only a rough guide to how well the JSC-Life 
Sciences Data Archive performed. Other elements of the total life sciences archive (NSSDC, Ames Research Center, and Mission Disk) (4) were also at the demonstration, and the questionnaire did not distinguish between the various elements. The different elements, however, use similar approaches and in some cases contain the same information (e.g. the NSSDC contains top level information from both JSC and ARC). Because of this we are confident that the questionnaires do provide a valuable initial look at how the JSC archive is received by potential scientific users.

\section{RESULTS}

Figure 4 shows the results from the questionnaire indicating overall reactions. Overall, the impression was favorable.

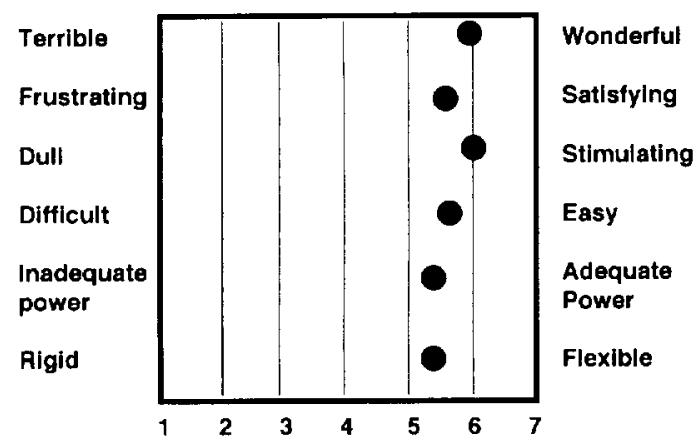

Figure 4. Questionnaire results.

Eighty-eight percent $(88 \%)$ of the individuals who completed a questionnaire said they would want to use the archive when it is completed. The reasons for wanting to use the archive were as follows (more than one answer could be chosen):

$82 \%$ To find background information on completed experiments

$71 \%$ To locate and browse experiment data

$48 \%$ To plan new experiments

$44 \%$ To combine data from more than one experiment or discipline

$22 \%$ To propose a data analysis or modeling project using archive data

Most evaluators felt they would need little instruction to use the archive. Eighty seven percent $(87 \%)$ said they would need either no training, a brief overview or a few pages on each of the major parts.

\section{CONCLUSION}

Acquiring data before, during and after spaceflight requires a major commitment of time and resources by investigators and NASA. These data need to be used to the fullest extent possible. The Johnson Space Center Life Sciences Data Archive prototype has been constructed to provide researchers, engineers, managers and educators interactive access to key life sciences data.

The initial evaluation of the archive has shown that the approach taken is wellreceived by scientists working in the field. Currently, the prototype is entering a formal evaluation phase. Privacy controls for the human data are also being devised. Once these tasks are complete the archive will be modified to meet user needs and expanded to include data from other missions.

\section{REFERENCES}

1. Beberness, BJ and Rosser, RS. Microsystems Integrated Real-time Acquisition Ground Equipment (MIRAGE) User's Guide for the Macintosh. NASA LS40066-1, 1994.

2. Duncan GT, Pearson RW. Enhancing access to microdata while protecting confidentiality: Prospects for the future. Statistical Science 6(3):219-239, 1991.

3. Johnston RS and Dietlein LF, eds. Biomedical Results from Skylab, Washington, DC: NASA, 1977.

4. Leonard JI, Callahan PX, Taylor G, Cardenas J, Mains R, King J, White RJ, Prototype of the Life Sciences Data Archive (Abstract), ASGSB

Bulletin 8(1), October 1994, p85

5. Maintaining the Privacy of Biomedical Research Data. NASA-Johnson Space Center Management Directive 1382.5 (1992). 
6. Marks VJ. User's guide for the Skylab Medical Data Analysis System. NASA CR160189, NASA-Johnson Space Center, Houston, 1975.

7. Policy Concerning Data Obtained from Space Sciences Flight Investigations. NASA Management Instruction 8030.3A (1978).

8. Privacy Act of 1974, 5 USC $\S 552 a$ (1992).

9. Rosser, RS and Rahman HH. MIRAGE: The data acquisition, analysis and display system. Proceedings of the third national technology transfer conference and exposition. NASA Conference Publication 3189, Vol. 2., pg. 419. 1992.

10. Space Act of 1958, 42 USC \$203a (1994).

11. Spross FR. Skylab Medical Data Center and Archives. NASA TM X-58148, NASAJohnson Space Center, Houston, 1974.

12. West JB. Life in space. J Appl Physiol. 72(5):1623-30. 1992. 